\title{
Personal and Shared Perspectives on Knowledge Maps in Learning Environments
}

\author{
Anna Goy, Giovanna Petrone, and Claudia Picardi ${ }^{(凶)}$ \\ Dipartimento di Informatica, Università di Torino, Torino, Italy \\ \{annamaria.goy, giovanna. petrone, \\ claudia.picardi\}@unito.it
}

\begin{abstract}
Knowledge maps are a powerful means to represent and share knowledge in both communication and learning. Collaborative knowledge mapping, in particular, enables comparing, discussing and bridging different perspectives on a topic. In this paper, we propose that it can be supported by providing users with multi-perspective maps, including one shared perspective and several individual ones. Building on our previous work about collaborative annotation of resources, we provide a formalization for multi-perspective concept maps, which we implemented in a proof-of-concept prototype. We then present the results of a formative qualitative evaluation performed on the prototype, where 12 participants, divided into 4 groups, performed a collaborative mapping task with two different versions of the tool: one in which only the shared perspective was available, and another in which the shared perspective was paired with a personal one. From the analysis of the observations gathered in the evaluation, as well as the subjective impressions of the participants collected by means of an electronic questionnaire, we draw requirements for an interaction model supporting multi-perspective concept maps. Such requirements can be summarized as follows: (1) the UI should overlay the personal and the shared perspective, to stress that they concern the same object (the map) and to enable comparison; (2) "shared" and "personal" should be supported by different work modalities, which should be explicitly enabled in the UI; (3) the UI should include a "revision of changes" mode to support users in evaluating changes by others, and relating their perspective to the work of others.
\end{abstract}

Keywords: Collaborative learning $\cdot$ Multi-perspective concept maps $\cdot$ Personal and shared perspectives

\section{Introduction and Related Work}

Knowledge maps - graphical representations aimed at "describing intellectual landscapes" [14] - are a powerful means to represent and share knowledge, with two major purposes:

- communicating/sharing knowledge with visual immediacy, making the relevant pieces of information and their connections easy to capture; 
- learning/acquiring knowledge; knowledge maps are in fact a widely recognized learning aid.

These two tasks are not independent from each other:

- communication is core to learning: teachers and students share their knowledge, and researchers have investigated the use of knowledge maps [3, 5, 12], in particular in collaborative learning [9];

- communication both implies and works toward mutual understanding: the receiver learns something from its source - not always new information, but certainly her counterpart's perspective.

In both communication and learning, a knowledge map can be seen as a perspective on a topic. Therefore, comparing, discussing and bridging different knowledge maps becomes relevant to the tasks of acquiring and sharing knowledge, as it allows to become aware of one's own map as a perspective, to be constructively critical towards the subjective aspects of maps, and to work towards the definition of a common, shared perspective.

Moreover, the development of a personal perspective is an important formative goal: supporting the learner in relating her personal knowledge map to the shared one and possibly to other learners' personal maps - can foster the development, recognition and meta-reflection on her own perspective [10].

In previous works, we have investigated how collaborative annotation of resources can be enhanced by keeping track of personal views [6, 7]. We found that the outcome of collaboration is perceived to be more satisfactory when each author is allowed to keep a personal view rather than sacrificing it to the common goal. Some users also reported that looking at the other participants' work on the shared view increased their awareness of their own perspective on the topic.

We have thus become convinced that the goal of collaboration is not necessarily to obtain an agreed-upon, univocal representation, but instead:

- to create awareness on the existence of different perspectives;

- to learn why, when and how one's own knowledge map represents a perspective rather than objective knowledge;

- to reach mutual understanding on the different perspectives;

- to learn from other people's perspectives, possibly modifying one's own as a result;

- to make available to others a multi-faceted, inclusive representation of "knowledge" on a given topic, which honors the different perspectives as well as the common grounds.

In order to implement this vision, and to be able to evaluate it, we applied it to a learning context characterized as: lifelong (a need that emerges from present-day knowledge-intensive and highly interconnected life styles [4]), ubiquitous (occurring anywhere, being open and loosely structured [8]), self-initiated and interest-driven (thus taking place outside formal education settings [2]).

Within this scenario, Personal Learning Environments (PLE) [1, 17] are emerging, allowing learners to build their own learning workspaces. Although PLEs often enable 
users to integrate shared and personal workspaces, the definition of models supporting these features is still an open issue [8].

The ultimate goal of our work is to define an interaction model, based on a formal representation of knowledge maps, coupled with automated techniques for comparing maps, highlighting similarities and differences with respect to different map features such as chosen concepts, types of connections and map topology [15].

In this paper, we take a first step in this direction, by drawing requirements, based on a formative qualitative evaluation, for an effective interaction model enabling users to handle both personal and shared perspectives within a web-based system for designing concept maps. Concept maps are a specific type of knowledge map [13], represented as connected graphs where nodes nodes represent ideas, thoughts, concepts, and edges represent connections between them.

In Sect. 2 we formally define multi-perspective concept maps; personal and shared perspectives are then characterized by the different actions that users can perform on them, along with the effects of such actions (Sect. 2.1). We then discuss the experimental scenario we envisioned (Sect. 2.2) and the proof-of-concept prototype we implemented in order to enable the evaluation (Sect. 2.3).

In Sect. 3 we present the results of a formative, qualitative evaluation of the interaction between users and multi-perspective concept maps; in Sect. 4 we discuss such results, outlining the lessons we learned from them, and drawing requirements for a novel proposal of interaction model. Section 5 concludes the paper highlighting future directions for research.

\section{Multi-perspective Concept Maps}

A concept map can be represented as a labelled graph $M=\left(C, R, l_{c}, l_{r}\right)$, where $C$ is the set of concepts represented in the map, $R \subseteq C \times C$ is the set of relationships among them, $l_{c}: C \longrightarrow \Sigma^{*}$ is the set of labels (on an alphabet $\Sigma$ ) used for concepts, and $l_{r}$ : $R \longrightarrow \Sigma^{*}$ is the set of labels used for relationships. Given such a map $M$ :

- A perspective $M^{\prime}$ on $M$ is a labelled subgraph $\left(C^{\prime}, R^{\prime}, l_{c}{ }^{\prime}, l_{r}\right)$, where $C^{\prime} \subseteq C, R^{\prime}$ is a subset of the projection of $R$ on $C^{\prime} \times C^{\prime}, l_{c}{ }^{\prime}$ is the projection of $l_{c}$ on $C^{\prime}$, and $l_{r}{ }^{\prime}$ is the projection of $l_{r}$ on $R^{\prime}$. In the following we will write $M^{\prime} \subseteq M$ to denote $M^{\prime}$ is a subgraph of $M$.

- Given a concept map $M$ collaboratively built by a group of users $\left\{u_{1}, \ldots, u_{n}\right\}$, for each user $u_{i}, P\left(u_{i}\right) \subseteq M$ denotes her personal perspective, while $P$ denotes the shared perspective.

- Each user $u$ can view and manipulate (add, delete, modify) those elements of $M$ (i.e., concept, relationships and labels) that belong to her $P(u)$. All users can view and manipulate elements of $M$ belonging to $P$. Manipulations on $P(u)$ affect only $P$ (u) itself. Manipulations on $P$ can affect also other $P(v)$ s, with $v \neq u$. Available actions and their effects on the different perspectives are described in detail in Sect. 2.1.

- In addition, a user $u$ can share elements belonging to her perspective $P(u)$, making them part of $P$ as well, or decide she disagrees with elements belonging to the shared perspective $P$, removing them from her $P(u)$. The effects of these actions are also detailed in Sect. 2.1. 


\subsection{User Activities}

The personal and shared perspectives are essentially characterized by (i) the actions that can be performed on them by the different users, and (ii) the effects these actions have on the other perspectives. These characterizations are an application of our previous work on collaborative tagging $[6,7]$ to multi-perspective concept maps.

A personal perspectives $P(u)$ can (and should) overlap with the shared perspective $P$ : their intersection contains those shared elements user $u$ agrees upon. On the other hand, the overlap between two personal perspectives $P(u)$ and $P(v)$ is contained in $P$; there cannot be common elements between two personal perspectives that are not shared among all. Of course, $P(u)$ and $P(v)$ can end up containing concepts or relationships with the same labels and connections, but they are regarded as different "objects" within the global map $M$.

A personal perspective $P(u)$ offers to its owner $u$, and only to her, the possibility to Add, Remove, Edit, Move, Resize and Share its "exclusive" elements, i.e. elements that belong only to $P(u)$. Of these actions, Share is the only one affecting other perspectives (namely, $P$ ). The shared perspective $P$ offers all users the possibility to Add, Remove, Edit, Move, and Resize all of its elements, or Disagree/Agree with them. Most of these actions affect in different ways the personal perspective of the acting user, and the personal perspectives of the others (Fig. 1).

- Add concept/relationship: this action performed in $P(u)$ has no effect on other perspectives $P(v) s$ nor in $\mathrm{P}$; if performed in $P$, the concept/relationship is added in $P$ (u) and to all the $P(v)$ s of other users, as participants are assumed by default to agree with it.

- Remove concept/relationship: this action can be performed in $P(u)$ only on elements that do not also belong to $P$; it has no effect on other personal perspectives. If performed in $P$, the element is removed from $P$ as well as from $P(u)$. Each $P(v)$, with $v \neq u$, containing the removed element, retains a copy of it belonging only to $P(v)$. In all cases, if the removed element is a concept/node, the action is applied in cascade to the relationships/edges connected to it in $P$ or $P(u)$.

- Edit concept/relationship editing includes both changing the label a of concept or relationship, or changing how a relationship is connected. This activity, if performed in $P(u)$, has no effect on other perspectives. If the action is performed in $P$, it affects all the perspectives containing the modified concept/relationship.

- Move/Resize concept: this activity, if performed on elements belonging only to $P$ $(u)$, has no effect on other perspectives. The position and size of elements belonging to $P$ can be changed only in the shared perspective, even if they also belong to $P(u)$. When this happens, the action affects all the perspectives containing the moved/resized concept/relationship.

- Share concept/: this activity is available only in the personal perspective, on concepts/relationship belonging $P(u)$ but not to $P$; its effect is to add the map element to $P$. If the shared element is a relationship/edge, and one or both the concepts/nodes it connects do not belong to $P$, they are also added in $P$. When an element is shared from $P(u)$ to $P$, we assume that, by default, it is added to the 
$P(v) s$ of other users $v \neq u$ too (in other words, we assume that other participants agree with it unless they explicitly disagree).

- Disagree with concept/relationship: this activity is available only in the shared perspective, and $u$ can only disagree with elements in $P$ that also belong to $P(u)$. The effect is to remove the element from $P(u)$; if the element is a concept also relationship/edges connected to it in $P(u)$ are removed.

- Agree with concept/relationship: this activity is available only in the shared perspective, and $u$ can only agree with elements in $P$ that do not belong to $P(u)$. The effect is to add the element to $P(u)$. If the element is a relationship, and one or both its connected concepts do not belong to $P(u)$, they are also added in $P(u)$, i.e. agreement is extended to them.

\subsection{Usage Scenario and User Interface}

The basic scenario we envision includes a small group of people (e.g., in our experiment, groups contain 3 users; see Sect. 3) collaborating in order to develop a shared concept map on a topic. Two variations can be devised (actually, two extremes in a spectrum of possibilities where the two situations are interleaved):

a. Users learn collaboratively by directly building a shared perspective. Personal perspectives essentially grow out of the shared one whenever there is a disagreement, i.e. a concept or relationship someone agrees with is removed by someone else.

b. Users learn first by themselves, building their personal perspectives, and only at a later stage, when they have reached a satisfactory conceptualization of what they have studied, they share their work. In this case the shared perspective emerges from a selective merge of the personal ones.

Here we focus on scenario (a), not because we deem irrelevant scenario (b), but in order not to broaden too much the scope of the study and risk creating confusion in the experimentation with users (see Sect. 3). In scenario (a) we therefore assume that users have the goal of building a final common map, and thus we expect the shared perspective to be their main focus. The characterization of user actions in the previous section takes into account the assumptions of scenario (a): whenever a user adds an element to the shared perspective, it is automatically added to the personal perspectives of all participants, and will be removed only if the will explicitly disagree with it.

\subsection{Proof-of-Concept Prototype}

Our proof-of-concept prototype, used in the evaluation described in Sect. 3, provides two versions of the tool for the collaborative management of concept maps: in the simpler version, named onlyS, users only interact with the shared perspective on the concept map; in the complete version, named SplusP, users can see and interact with both their personal perspectives and with the shared one, shown side-by -side on the screen (Fig. 1). 
On the left, the user can see and interact with the personal perspective, while on the right she can see and act on the shared perspective. At any time, only one of the two is active, highlighted by a white background (the inactive one is grey). The user makes a perspective active by clicking on it.

In the personal perspective, white nodes represent exclusively personal concepts, while grey ones are shared nodes the user has agreed with. The former can thus be freely edited, moved or resized, while the latter can be edited, moved or resized only from the shared perspective. The current user interface does not make any graphical distinction between shared/non-shared agreed/non-agreed relations.

The user double clicks on the canvas to Add a new concept, while she double clicks on an existing element to Edit it (when this action is available). To Add a relationship between two concepts, the user selects a concept, clicks on "Aggiungi relazione" (Add relationship) button and then clicks on the second concept. Concepts can be Removed by first clicking on them and then pressing Backspace. A user can indeed do so with a shared concept in the personal perspective, but this triggers the Disagree action (the shared concept is removed from the personal perspective but retained in the shared one.

Concepts can be Moved or Resized by dragging either the concept itself or the resize handles, as in any shape drawing UI.

If the user in the personal perspective wants to Share a concept, she selects the concept and clicks on "Condividi con altri" (Share with others) button.

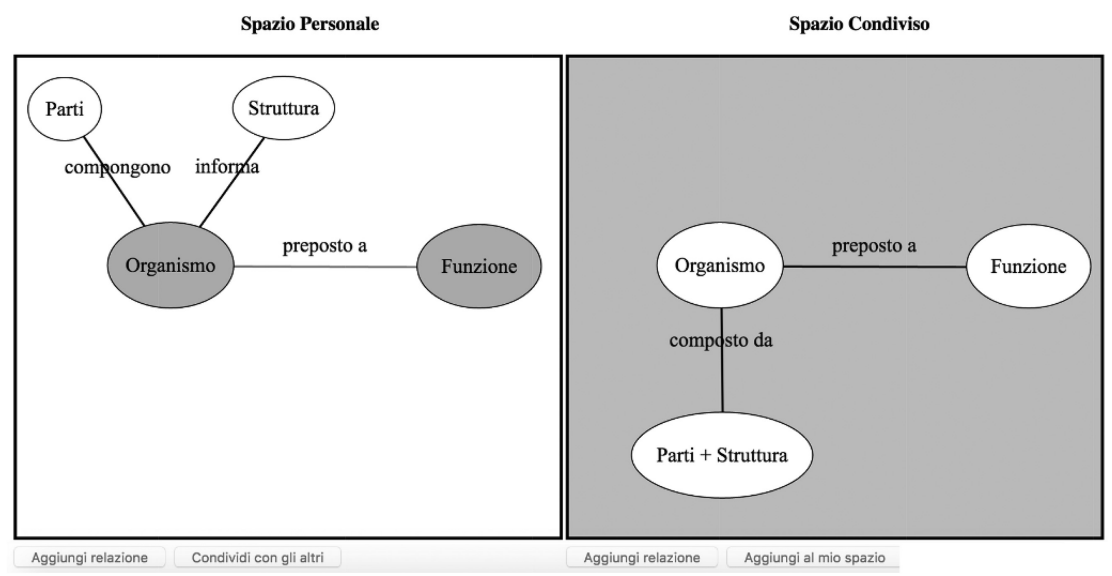

Fig. 1. Screenshot of the SplusP prototype

In the shared perspective, the user can also Agree again with a concept she had previously disagreed with, thereby re-adding it to her personal perspective; this can be obtained by selecting the concept and clicking on the button labelled "Aggiungi al mio spazio" (Add to my space). 
The prototype has been built as a Web Application, exploiting in the front-end HTML5, JQuery and FabricJS, which is a specialized library to draw on canvas. The backend is implemented in PHP and MySQL. Our goal has been to quickly test the approach and be able to modify the user interaction, based on the user evaluation we performed on the current prototype.

\section{Qualitative Evaluation}

\subsection{Assumptions}

Type of collaboration. Our investigation broadly concerns collaboration in building concept maps. However, this includes a wide range of sub-tasks and situations involving different dynamics both in the user-to-user interaction and in the user-to-map interaction.

We can trace two major distinctions in collaborative work $[11,16]$ :

- colocated vs. remote

- synchronous vs. asynchronous

With respect to these distinctions, which result in four quite different types of collaborative activity, we narrowed down our study to the case of remote, asynchronous collaboration. Therefore, we made the following assumptions:

- People work on the same document (in our case a concept map) but they do not do it at the same time; the document is available on a shared repository but only one person at a time can modify it.

- People cannot communicate with voice or gesture, or other visual or aural cues.

Task. Referring to the two scenarios envisioned in Sect. 2.2 (points (a) and (b)), in order to avoid too much variability in our experiments, we chose to focus on scenario (a), at least for what concerns the formulation of the assignment, i.e., participants directly work on building a shared map; personal perspectives essentially grow out of the shared one whenever there is a disagreement.

Focus of observation. We chose to focus on observing:

- How the participants interacted with the shared concept map, i.e. the type of actions they carried out on map elements both created by them or by others.

- How the participants interacted with the personal perspective, when available.

- The participants' reactions to what happened during the collaboration.

We did not focus on the cognitive task of building the map per-se, and we did not observe nor address the semantic content of the resulting concept maps. 


\subsection{Experimental Setup}

We recruited 12 participants ${ }^{1}$ which were then divided into 4 groups (G1, G2, G3 and G4) of 3 people each. Each group received an initial briefing, describing the simulated task they had to carry out: "You are studying for an introductory psychology course, and you received a group assignment. You have to collaborate at building a concept map representing the relevant notions of a book section, and you need to do so using the digital tool we will provide you with."

In order to simulating a remote, asynchronous collaboration, users cannot communicate with each other during the task.

Each participant was provided with a short excerpt from an introductory psychology book; after reading it, each participant took two turns (lasting no more than ten minutes) at the computer.

Each group underwent the experiment twice, with two different text excerpts (X and Y), and with two different versions of the tool: onlyS, in which only the shared perspective was available, and Splus $\boldsymbol{P}$, in which the shared perspective on the map was coupled with a personal one. Groups performed the experiment according to the following layout:

\begin{tabular}{l|l|l}
\hline & First onlyS then SplusP & First SplusP then onlyS \\
\hline First X then Y & G1 & G3 \\
\hline First Y then X & G2 & G4 \\
\hline
\end{tabular}

One of us took notes recording the comments that the person using the tool voiced out loud while working, as well as their overall approach to the work, and any obstacle, difficulty or issue that could arise in the interaction. The recording of specific actions within the tool was carried out by the logging service of the application.

For each group, after both experiments were completed, we collected each participants' feedback by means of a computer-based questionnaire, described in the next section.

\subsection{Results}

Interaction Dynamics. We recorded (by taking notes and by logging user actions) the dynamics of the activity for each group (G1, G2, G3) with each tool (onlyS or SplusP). We also collected the comments of the users during the interaction, because it gives a better feel of the context where they were voiced. The dynamics of the activity as well as the users' comments are reported in Appendix A.

Table 1 shows the number of logged actions, by type, carried out across the whole experiment, either in the shared or in the personal space (when available).

\footnotetext{
${ }^{1}$ We recruited the participants among students in our university, with varied backgrounds either in computer science, or human studies.
} 
Table 1. Action types across all groups

\begin{tabular}{|c|c|c|c|c|c|c|c|c|c|c|c|c|c|c|}
\hline & \multirow{2}{*}{$\begin{array}{l}\text { tot } \\
\text { azioni }\end{array}$} & \multicolumn{3}{|c|}{ add/edit/delete } & \multirow{2}{*}{ layout } & \multirow[t]{2}{*}{ add } & \multicolumn{3}{|l|}{ edit } & \multicolumn{3}{|c|}{ delete } & \multirow{2}{*}{$\begin{array}{l}\text { share } \\
\text { own }\end{array}$} & \multirow{2}{*}{$\begin{array}{l}\text { copy to } \\
\text { pers. }\end{array}$} \\
\hline & & tot & own & other & & & own & other & tot & own & other & tot & & \\
\hline onlyS & 427 & 203 & 155 & 48 & 224 & 143 & 8 & 20 & 28 & 4 & 28 & 32 & - & - \\
\hline $\begin{array}{l}\text { SplusP } \\
\text { personal }\end{array}$ & 186 & 118 & - & - & 68 & 54 & - & - & 1 & - & - & 63 & - & - \\
\hline $\begin{array}{l}\text { SplusP } \\
\text { shared }\end{array}$ & 381 & 137 & 116 & 21 & 215 & 105 & 5 & 8 & 13 & 6 & 13 & 19 & 28 & 1 \\
\hline $\begin{array}{l}\text { SplusP } \\
\text { total }\end{array}$ & 567 & 255 & 116 & 21 & 283 & 159 & 5 & 8 & 14 & 6 & 13 & 82 & 28 & 1 \\
\hline
\end{tabular}

Questionnaire. The goal of the questionnaire was to obtain the subjective evaluation of the participants concerning the task we asked them to complete, and the tools they used to do so. The questionnaire was composed of 5 questions and a space for free final comments. We list here the 5 questions and provide histograms representing the participants' answers (Fig. 2). Free comments are fully listed in Appendix B; pertinent remarks are also quoted in the discussion (Sect. 5).

Q1: Given your previous experience (if any) and what you did today, how does it seem to you that drawing knowledge maps, either on paper or with a computer-based tool, as an aid to studying and learning, is easy/interesting/useful? (answers on a 5-points rating scale)

Q2: Which of the two tools you experimented with today was easier/more interesting/more useful for drawing knowledge maps as an aid to studying and learning? (answers on a 5-points rating scale)

Q3: Having a personal space in addition to the shared one was useful to you for... (multiple choice with open option)

Q4: According to the experience you had today, which of the following additional features could improve the tool(s)? (multiple choice)

Q5: Are there any other additional features or changes you would suggest for the SplusP tool? (free text)

\section{Discussion and Requirements}

Splus $P$ was found by almost half the participants less easy to use than onlyP. The choice of tool did not seem to make the task more or less interesting; participants were divided on whether SplusP was more or less useful than onlyP. However, all participants but one put the personal perspective to use (see answers to questionnaire Q3). The overall analysis of the observations, including interaction dynamics, action log, and questionnaires, shows in our opinion that, in most cases, what the participants subjectively reported in the questionnaire is supported by our direct observation of their interaction.

In particular, our analysis shows that while users perceived the personal perspective feature as potentially interesting, the way it was implemented let their expectations 


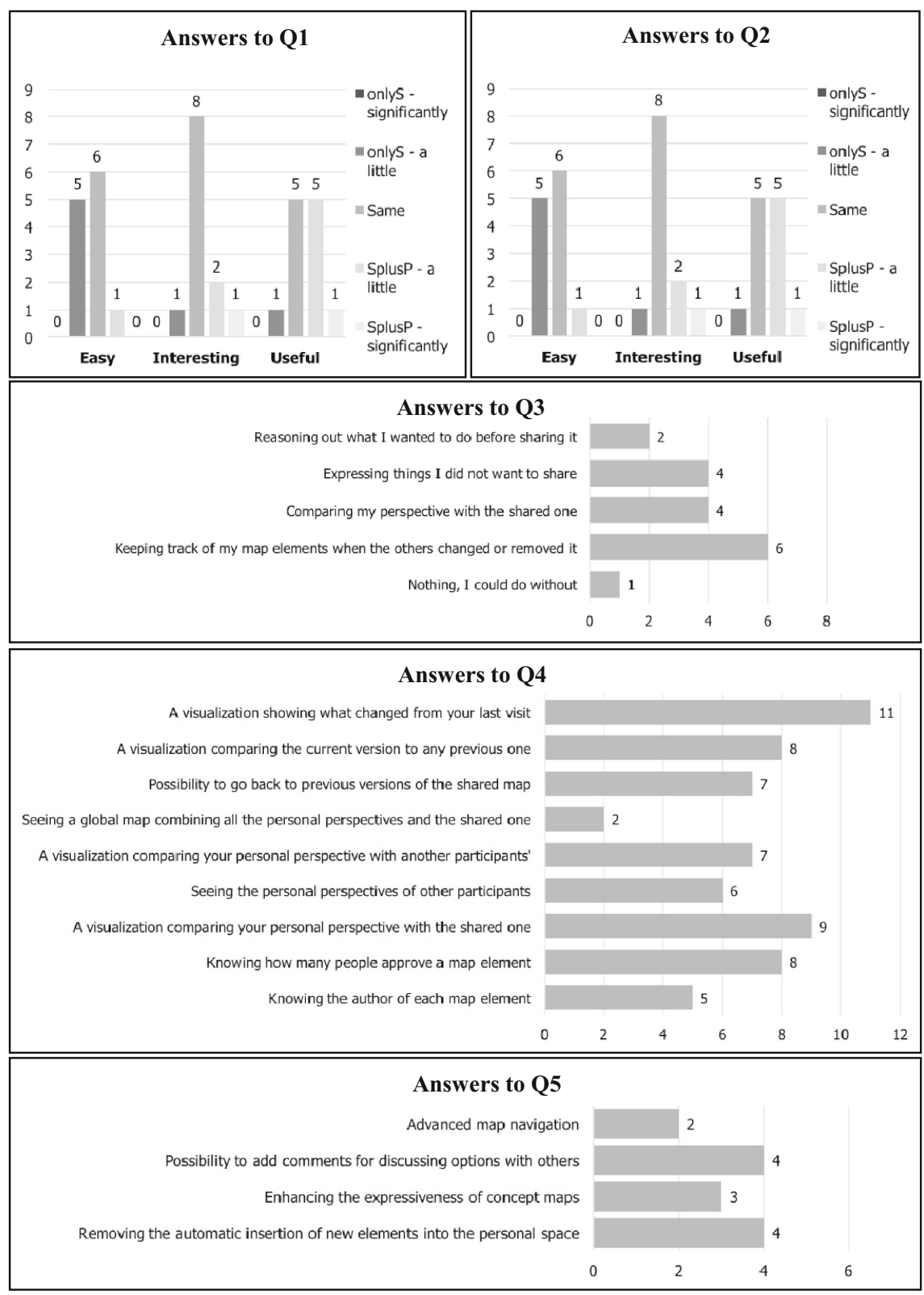

Fig. 2. Answers to questionnaire

down. Converging towards a shared perspective was deemed a difficult task in most difficulties attained to the task per-se, which were caused by interaction problems, and which could be eased by additional functionalities. 
In the following we address the issues that were more frequently reported or encountered during the interaction.

Participants perceived the personal perspective as a repository of map elements rather than as a way to highlight and keep track of their point of view. Some users never or seldom considered their personal space. Most actions within the personal space were performed in one of these two situations:

- The user had just started working and used the personal perspective as a "sketch book" to devise a "good" map to be later shared (about 1 participant out of 4).

- The user was on her second round; she checked out the personal space, comparing it with the shared one in order to see if her work had been kept or removed by the other participants (about half the participants).

Some perceived the personal perspective as being too separate, both spatially and conceptually, from the shared perspective: "I liked the idea of having a personal space, but I think it did not work really well having it on the side, as if it was a separate thing"; "I would have liked to use more the personal space, but I found that it was too separate from the shared space, and our task was to build a shared perspective". The rest of the analysis provides additional insights on this perception of "separation".

Participants found it difficult to "read" the map fragments provided by others, and to understand the others' perspective when different from their own. If we look at the observed behaviour and at the performed actions, we see that very few people actually tried to bridge their work with what others had done. Some of them did not feel they could work at all on the shared map, because they "could not find how to bridge the differences" and "would need to reorganize [it] completely", to use their own words. Of the 12 participants, 4 people recommended, as additional feature, the possibility of adding verbal comments to the map, while other 3 asked for enhancements to the mapping language (e.g., directed arrows for edges), which they found lacking in terms of expressiveness.

Most participants worked by adding their map fragment to the shared perspective, restating with different wording and structure what had already been expressed, and without actually being able to relate their fragment to what already present. They did not feel supported in relating the two perspectives by the availability of a personal space, because, as one of them remarked in the questionnaire, "it did not help me find similarities or differences". Others echoed the feeling: "I felt it was not so easy to see what was different between the personal and the shared space", "...thinking that I would have the possibility to compare [the personal space] with what the others did, but then I found that it was not really possible". Unsurprisingly, 9 of the 12 participants said in the questionnaire that a visualization highlighting differences between the personal and the shared perspective would be appreciated.

We saw that adding by default new map elements to the personal space of every participant, when such elements were added to the shared space, was a poor choice. Our idea was that, given the goal of a shared map, people would have tried to build upon the others' work, even in their personal space. Also, we thought that in this way everyone would be compelled to see what had been added from her last visit, and take a stance by either keeping it or discarding it from the personal perspective. However, most participants actually saw this as a defilement of their personal space: "the shared space turned out a bit cluttered"; "[in the personal space] I had no means to 
distinguish the others' work from mine"; "I was not interested in keeping what the others did in [my personal space]"; "I... did not look much at the personal [space]. Also because it got cluttered with the others' work".

All participants but one (11 people out of 12) said they would recommend, as an additional feature, the possibility of seeing changes with respect to their last visit. There seem to be two main reasons for this, according to their free remarks: on the one hand, to make clearer the proposed change, while still leaving room for undoing it: "I still had concerns about removing the others' work. ... if I removed what the others did, they maybe would not realize it"; "I was uncomfortable in removing things from the shared space, because it seemed to be rude. I would like to be able to suggest a removal, then let the others decide"; "I think it would be more useful for the collaboration to be able to see the changes proposed by each person". On the other hand, highlighting newly added elements allows to cherry pick what to add in one's own perspective and what to discard. As one participant put it: "I did not like the fact that the others' work was by default added to my personal space. I would have liked to be able to distinguish what was "new" from the last time I worked on it."

\subsection{Improving the Interaction}

Our present goal, given the above discussion of experimental results, is to propose a different interaction modality for supporting users in working with multi-perspective concept maps. However, the experiment showed that the focus should be shifted from the task of designing a shared concept map, to the task of comparing and reasoning on the different perspectives. Without achieving this goal, it is impossible for the group to build a shared perspective which honours the individual points of view.

The interaction modality implemented in the prototype was not up to this task: it brought users to see the personal perspective as a temporary, private repository, rather than as a way to clarify a participant's point of view. Nonetheless, the prototype allowed the participants to experiment interaction with both a personal and a shared perspective, which in turn provided us with insights on how such interaction could be improved.

There are two major points that the analysis of the experimental observations made clearer to us:

- Perspectives arise when we look at the same object from different points of view. Even if we see different parts or aspects of the object, the object is the same. Our participants reminded us of this when complaining about having two separate screen spaces, and when expressing the desire for a differential visualization.

- "Personal" and "shared" do not only denote different perspectives on the same object, but also different work modalities on the same object. Working from a given perspective is different than working on a different perspective. When a person works from a personal perspective, she acts on its elements, but her actions affect also the shared perspective. The opposite is also true. Our prototype did not clearly highlight this distinction, nor provide adequate feedback on the effects of users' actions, so that they could be supported in recognizing the distinction. 
Based on these considerations, and on the analysis in Sect. 4, we propose that a novel interaction model for multi-perspective concept maps should take into account the following requirements:

1. The visualization should overlay the personal and the shared perspective. The visual cues communicating if a map element belongs or not to each perspective should be independent from each other: it should be immediately distinguishable if a map element belongs to the personal perspective, to the shared, or both.

2. The user should be able to choose between two different editing modalities, "personal" and "shared". It should be clear that in each modality the user essentially acts from the corresponding perspective, but her actions can affect also the other one (having both perspectives visible in overlay should help stressing this point). Strong visual cues should clearly communicate what modality is currently active.

3. Changes since last visit can be visualized too as an overlay between two (shared) perspectives: the last one the user worked with, and the new one that evolved while she was offline. This suggests that the "shared" modality mentioned in the previous point could include a "revision of changes" activity or work mode, supporting the possibility, mentioned by some of our participants, of expressing a vote or opinion on a suggested change. A revision of changes would also solve the problem of whether new elements added in the shared perspective should be automatically agreed with or not (as we saw, agreement by default was not appreciated by the evaluation participants). By specifically revising newly added concepts and relationships, the user would be supported in choosing what to do with each.

\section{Conclusions}

In this paper, we introduced multi-perspective concept maps as a tool for learning through collaboration in building concept maps for a given subject of study.

We defined the notion of perspective on a concept map, and we characterized what a personal and a shared perspective are in a group collaboration, by describing the different actions that each user can perform on the two types of perspectives, and the effects such actions have on the others' work. These definitions built upon our previous work on multiple perspectives in collaborative annotation of resources $[6,7]$.

We described a proof-of-concept prototype we implemented to test our proposal. We then presented the results of a formative qualitative evaluation, discussing them in order to draw principles and requirements for an effective interaction model for users to collaboratively work with both a shared and a personal perspective.

These principles and requirements also form the starting point for our future work on this topic. Our immediate goal is to design, and thoroughly evaluate, a new prototype incorporating the requirements emerged from the user study: overlay visualization of perspectives, multiple editing modalities, and a "revision of changes" feature. According to the users' feedback, the new prototype should also incorporate the possibility to discuss changes and choices with group members, by means of a commenting feature, and offer an enriched concept mapping language to allow for more expressiveness. 


\section{Appendix A}

Here we fully describe the interaction dynamics observed during the evaluation.

P1, P2, P3 refer to the different participants in each group (Tables 2, 3, 4, 5, 6, 7, 8 and 9).

Table 2. Interaction dynamics for G1, Tool onlyS

\begin{tabular}{l|l}
\hline All & Read text printed on paper \\
\hline All & Sketch out a possible concept map on the text sheet \\
\hline P1, round 1 & Recreates the map sketched out on paper \\
\hline P2, round 1 & $\begin{array}{l}\text { Does not touch what P1 did. Adds her own version of map } \\
\text { "The screen is small" }\end{array}$ \\
\hline P3, round 1 & $\begin{array}{l}\text { Essentially moves around the others' nodes, adding a few of her own } \\
\text { "I don't get it" } \\
\text { "I would like to remove everything the others did, is that possible?" }\end{array}$ \\
\hline P2, round 2 & $\begin{array}{l}\text { Reorganizes the map, removing a few of the other participants' concepts } \\
\text { "Is there a scrollbar?" (there is none: users have to drag the canvas in order to } \\
\text { scroll) }\end{array}$ \\
\hline P3, round 2 & $\begin{array}{l}\text { Takes on what exists (deleting and modifying other participants' work) and } \\
\text { relationships }\end{array}$ \\
\hline
\end{tabular}

Table 3. Interaction dynamics for G1, Tool SplusP

\begin{tabular}{l|l}
\hline All & Read text printed on paper \\
\hline All & Sketch out a possible concept map on the text sheet \\
\hline P3, round 1 & $\begin{array}{l}\text { Recreates the map sketched out on paper in the personal space. Tries to share } \\
\text { it all at once, failing (the tool does not allow it). Shares part of the map } \\
\text { "I will decide what to do with the rest after the others have done their part" }\end{array}$ \\
\hline P1, round 1 & Works exclusively in the shared space, adding her own map to it \\
\hline P2, round 1 & $\begin{array}{l}\text { Works a little bit (moving, adding) in the personal space but without sharing } \\
\text { anything. Works a little bit (again moving, adding) in the shared space } \\
\text { "I would need to reorganize this completely" }\end{array}$ \\
\hline P3, round 2 & $\begin{array}{l}\text { Removes concepts from the personal space. Also, reorganizes the shared } \\
\text { space, moving, editing and deleting what the others did }\end{array}$ \\
\hline P1, round 2 & $\begin{array}{l}\text { Again, works exclusively in the shared space, and only adds concepts and } \\
\text { relationships }\end{array}$ \\
\hline P2, round 2 & $\begin{array}{l}\text { Works only in the personal space, removing concepts and relationships, and } \\
\text { adding new ones }\end{array}$ \\
\hline
\end{tabular}


Table 4. Interaction dynamics for G2, Tool onlyS

\begin{tabular}{|c|c|}
\hline All & Read text printed on paper \\
\hline All & Take notes but do not sketch out the map on paper \\
\hline $\mathrm{P} 1$, round 1 & $\begin{array}{l}\text { Starts to create her map directly on screen. Adds a few concepts and } \\
\text { relationships. Appears uncertain }\end{array}$ \\
\hline $\mathrm{P} 2$, round 1 & $\begin{array}{l}\text { Adds her own version of the map, without deleting anything. Edits a couple of } \\
\text { P1's concepts } \\
\text { "If I had more time I would delete something" }\end{array}$ \\
\hline $\mathrm{P} 3$, round 1 & $\begin{array}{l}\text { Adds some concepts and relationships. and moves the existing ones. Deletes a } \\
\text { few of the other participants' map elements }\end{array}$ \\
\hline $\mathrm{P} 1$, round 2 & Deletes some of the existing concepts and adds some other of her own \\
\hline $\mathrm{P} 2$, round 2 & $\begin{array}{l}\text { Significantly edits the existing map (making changes and deleting). Also, adds } \\
\text { a few elements }\end{array}$ \\
\hline $\mathrm{P} 3$, round 2 & $\begin{array}{l}\text { Only edits and deletes a few elements, without reorganizing the space } \\
\text { "I would like to add a reflexive relationship" }\end{array}$ \\
\hline
\end{tabular}

Table 5. Interaction dynamics for G2, Tool SplusP

\begin{tabular}{l|l}
\hline All & Read text printed on paper \\
\hline All & Take notes but do not sketch out the map on paper \\
\hline P3, round 1 & $\begin{array}{l}\text { Adds a few concepts and relationships in both personal and shared space. } \\
\text { Then edits and deletes some (from both spaces) }\end{array}$ \\
\hline P1, round 1 & Adds her own concepts and relationships in the shared space \\
\hline P2, round 1 & $\begin{array}{l}\text { Works at reorganizing the shared space changing the map layout. Adds a few } \\
\text { concepts/relationships of her own, and edits a couple of the existing ones }\end{array}$ \\
\hline P3, round 2 & $\begin{array}{l}\text { Cleans out the personal space from concepts and relationships she is not } \\
\text { interested in. Adds a few concepts/relationships in the shared space }\end{array}$ \\
\hline P1, round 2 & $\begin{array}{l}\text { Works mostly at reorganizing the personal space, deleting unwanted } \\
\text { concepts/relationships and adding new ones } \\
\text { "The shared space is a lot tidier than my own" }\end{array}$ \\
\hline P2, round 2 & $\begin{array}{l}\text { Again, reorganizes the shared space, adding a few map elements. Does not } \\
\text { consider the personal space }\end{array}$ \\
\hline
\end{tabular}

Table 6. Interaction dynamics for G3, Tool SplusP

\begin{tabular}{l|l}
\hline All & Read text printed on paper \\
\hline All & $\begin{array}{l}\text { P1 and P2 sketch out the map on paper. P3 underlines significant concepts or } \\
\text { phrases in the text }\end{array}$ \\
\hline P1, round 1 & $\begin{array}{l}\text { Recreates the map she sketched on paper in the shared space } \\
\text { "I would like to be able to add a one-to-many relationship" }\end{array}$ \\
\hline P2, round 1 & $\begin{array}{l}\text { Adds her own concepts and relationships in the shared space } \\
\text { "The personal space does not seem useful in the first round" }\end{array}$ \\
\hline P3, round 1 & $\begin{array}{l}\text { Adds her own concepts and relationships in the shared space } \\
\text { "I do not like that there is stuff in my personal space that I did not put there" }\end{array}$ \\
\hline
\end{tabular}


Table 6. (continued)

\begin{tabular}{l|l}
\hline P1, round 2 & $\begin{array}{l}\text { Rearranges the layout in the shared space and adds a few } \\
\text { concepts/relationships }\end{array}$ \\
\hline P2-, round 2 & $\begin{array}{l}\text { Removes concepts/relationships from both the spaces. Adds a few elements } \\
\text { to the shared space }\end{array}$ \\
\hline P3, round 2 & $\begin{array}{l}\text { Adds a couple of elements to the personal space } \\
\text { "I do not really understand what the others did" }\end{array}$ \\
\hline
\end{tabular}

Table 7. Interaction dynamics for G3, Tool onlyS

\begin{tabular}{l|l}
\hline All & Read text printed on paper \\
\hline All & $\begin{array}{l}\text { P1 and P2 sketch out the map on paper. P3 underlines significant concepts or } \\
\text { phrases in the text }\end{array}$ \\
\hline P3, round 1 & $\begin{array}{l}\text { Adds a few concepts/relationships } \\
\text { "Before doing too much I want to see what the others do" }\end{array}$ \\
\hline P1, round 1 & Edits all the existing elements and adds many of her own \\
\hline P2, round 1 & $\begin{array}{l}\text { Slightly changes the layout of the map } \\
\text { "For me the map is ok as they did it" }\end{array}$ \\
\hline P3, round 2 & Mostly changes the layout, adding only a couple of elements \\
\hline P1, round 2 & Mostly changes the layout \\
\hline P2, round 2 & Adds a couple of elements \\
\hline
\end{tabular}

Table 8. Interaction dynamics for G4, Tool SplusP

\begin{tabular}{l|l}
\hline All & Read text printed on paper \\
\hline All & Take notes but do not sketch out the map on paper \\
\hline $\mathrm{P} 1$, round 1 & $\begin{array}{l}\text { Starts creating her map in the personal space } \\
\text { "I expected to be able to share it all at once" (But it is not possible). She then } \\
\text { chooses, given the time constraints, to wait for the next round }\end{array}$ \\
\hline $\mathrm{P}$, round 1 & $\begin{array}{l}\text { Adds map elements to the shared space. Then adds some other elements also } \\
\text { to the personal space }\end{array}$ \\
\hline $\mathrm{P} 3$, round 1 & $\begin{array}{l}\text { Adds map elements to the personal space, then shares some of them to the } \\
\text { shared space } \\
\text { "I would like to be able to express a one-to-many relationship" }\end{array}$ \\
\hline $\mathrm{P}$ 2, round 2 & $\begin{array}{l}\text { Reorganizes the map layout in the shared space, without adding or deleting } \\
\text { anything } \\
\text { "I would like to add a relationship between a concept and a relationship" }\end{array}$ \\
\hline P3, round 2 & $\begin{array}{l}\text { Mostly reorganizes the map layout in the shared space. Adds a couple of map } \\
\text { elements to the shared space } \\
\text { without changing anything } \\
\text { "My personal space is too messed up; I cannot compare my perspective to the } \\
\text { shared one" }\end{array}$ \\
\hline
\end{tabular}


Table 9. Interaction dynamics for G4, Tool onlyS

\begin{tabular}{l|l}
\hline All & Read text printed on paper \\
\hline All & Take notes but do not sketch out the map on paper \\
\hline P3, round 1 & Builds her own map, adding concepts and relationships \\
\hline P1, round 1 & Rearranges the map, editing a few elements. Adds a new concept \\
\hline P2, round 1 & Adds her own version of the map, without removing the existing elements \\
\hline P3, round 2 & Edits a few elements added by the others, and rearranges the layout \\
\hline P1, round 2 & Mostly rearranges the map layout, adding a couple of elements \\
\hline P2, round 2 & $\begin{array}{l}\text { Adds several elements to the map, and rearranges the layout, without } \\
\text { removing or editing anything }\end{array}$ \\
\hline
\end{tabular}

\section{Appendix B}

Here we report the complete free remarks the participants provided us with in the questionnaire (Table 10).

Table 10. Participants' answers to the final question (free comments)

a I had problems with the position of the concepts on the screen. The other participants preferred to organize concepts in a star-shaped layout, with the most relevant concept in the centre, while I prefer a tree-like structure, with the most relevant concept at the top. I also had some difficulties with the interface, it was not so easy to arrange the map

b I found it difficult to merge my own idea of concept map with the others; I could not read really well what the others had in mind, as if the map itself did not give me enough information. Also, I am not sure my perspective could really be bridged to the others, I felt the need to discuss my perspective with them before taking any actions, or we would end up bouncing between different perspectives depending on who had the last word. (Did you take advantage of the personal space?) I did not think the personal space would help me with this, because it did not help me find similarities or differences. And I thought that since our task was to build a shared perspective, working on my personal one would be a loss of time

c I had difficulty to understand the reasons behind the others' actions, then when it was my turn I would have preferred to re-start from scratch. Having a personal space did not make it easier, I still had concerns about removing the others' work. Maybe because I felt it was not so easy to see what was different between the personal and the shared space. As a consequence, if I removed what the others did, they maybe would not realize it, and, in any case, they would not understand while I did it. I certainly felt so when they removed my work! I mean, I could not understand why

d I did not like the fact that the others' work was by default added to my personal space. I would have liked to be able to distinguish what was "new" from the last time I worked on it. As it is, I spent a lot of time removing unwanted stuff from my personal space, which in the end did not turn out to be useful in building the shared perspective. At a certain point, I thought I would have preferred to work on my personal space alone, and then discuss with the others showing them my perspective and seeing theirs, before trying to come up with a shared version 
Table 10. (continued)

e Honestly, I did not find the personal space to be really useful, and I almost did not use it. I think it would be more useful for the collaboration to be able to see the changes proposed by each person, like the different versions of the map

f Every time I clearly saw the two perspective of the other two participants put together in the shared map, and I tried to sort them out, taking into account also my perspective. I liked the idea of having a personal space, but I think it did not work really well having it on the side, as if it was a separate thing

g I think it may have been useful to be able to see the others' personal spaces, to better understand what they had in mind. The shared space turned out a bit cluttered

$\mathrm{h}$ I was uncomfortable in removing things from the shared space, because it seemed to be rude. I would like to be able to suggest a removal, then let the others decide. In general I would have liked to be able to communicate with the other participants beyond the work we did on the map

i My personal perspective was completely different from what the others expressed. I could not find how to bridge the differences. (What about the personal space?) It did not feel like the solution to this problem. Also, I did not like to find in it stuff before I had even accessed it once

j It wasn't easy to understand the others' maps, this type of maps is too simple and leaves a lot of room to interpretations. Also, I spent a lot of time working on my personal perspective, thinking that I would have the possibility to compare it with what the others did, but then I found that it was not really possible, I had no means to distinguish the others' work from mine, it was a bit like those games where you have to find the differences between two quasi-identical images. It would be useful to be able to leave notes or suggestions for the others

$\mathrm{k}$ I mostly put in the shared map my own version, without working on what the others did, apart from rearranging it. I used the personal space as a notebook for thinking, I was not interested in keeping what the others did in it

1 I would have liked to use more the personal space, but I found that it was too separate from the shared space, and our task was to build a shared perspective. So, I began by using the personal space, but then I moved to work on the shared space and did not look much at the personal one. Also, because it got cluttered with the others' work

\section{References}

1. Attwell, G.: Personal learning environments - the future of eLearning? eLearning Pap. 2(1), 1-7 (2007)

2. Barron, B.: Interest and self-sustained learning as catalysts of development: a learning ecologies perspective. Hum. Dev. 49, 193-224 (2006)

3. Basque, J., Paquette, G., Pudelko, B., Leonard, M.: Collaborative knowledge modelling with a graphical knowledge representation tool: a strategy to support the transfer of expertise in organisations. In: Okada, A., Buckingham Shum, S.J., Sherborne, T. (eds.) Knowledge Cartography. Advanced Information and Knowledge Processing, pp. 491-517. Springer, London (2014)

4. Billett, S.: The workplace as learning environment: introduction. Int. J. Educ. Res. 47(4), 209-212 (2008) 
5. Draper, D.C.: Collaborative instructional strategies to enhance knowledge convergence. Am. J. Distance Educ. 29(2), 109-125 (2015)

6. Goy, A., Magro, D., Petrone, G., Picardi, C., Rovera, M., Segnan, M.: An integrated support to collaborative semantic annotation. Adv. Hum. Comput. Interact. 2017, 12 (2017). doi:10.1155/2017/7219098. Article ID 7219098

7. Goy, A., Magro, D., Petrone, G., Picardi, C., Segnan, M.: Shared and personal views on collaborative semantic tables. In: Molli, P., Breslin, J.G., Vidal, M.-E. (eds.) SWCS 2013-2014. LNCS, vol. 9507, pp. 13-32. Springer, Cham (2016). doi:10.1007/978-3-31932667-2_2

8. Häkkinen, P., Hämäläinen, R.: Shared and personal learning spaces: challenges for pedagogical design. Internet High. Educ. 15, 231-236 (2012)

9. Hmelo-Silver, C.E., Chinn, C.A., Chan, C., O’Donnell, A.M.: The International Handbook of Collaborative Learning. Routledge, London (2013)

10. Kandiko, C., Hay, D., Weller, S.: Concept mapping in the humanities to facilitate reflection: externalizing the relationship between public and personal learning. Arts Humanit. High. Educ. 12(1), 70-87 (2012)

11. Johansen, R.: GroupWare: Computer Support for Business Teams. The Free Press, New York (1988)

12. Molinari G.: From learners' concept maps of their similar or complementary prior knowledge to collaborative concept map: dual eye-tracking and concept map analyses. Psychologie française (2015). doi:10.1016/j.psfr.2015.11.001

13. Novak, J.D.: Learning, Creating and Using Knowledge: Concept Maps as Facilitative Tools in Schools and Corporations, 2nd edn. Routledge, London (2010)

14. Okada, A., Buckingham Shum, S.J., Sherborne, T.: Knowledge Cartography: Software Tools and Mapping Techniques, 2nd edn. Springer, London (2014)

15. Schwendimann, B.A.: Making Sense of Knowledge Integration Maps. In: Ifenthaler, D., Hanewald, R. (eds.) Digital Knowledge Maps in Education, pp. 17-40. Springer, New York (2014)

16. Skaf-Molli, H., Ignat, C., Rahhal, C., Molli, P.: New work modes for collaborative writing. In: Granville, B., Kutti, N.S., Missikoff, M., Nguyen, N.T. (eds.) Proceedings of International Conference on Enterprise Information Systems and Web Technologies, EISWT-2007, Orlando, United States, pp. 176-182 (2007)

17. Wilson, S., Liber, O., Johnson, M., Beauvoir, P., Sharples, P., Milligan, C.: Personal learning environments: challenging the dominant design of educational systems. J. e-Learn. Knowl. Soc. 3(2), 27-38 (2007) 